NASA/CR-2006-213925

NIA Report No. 2005-07
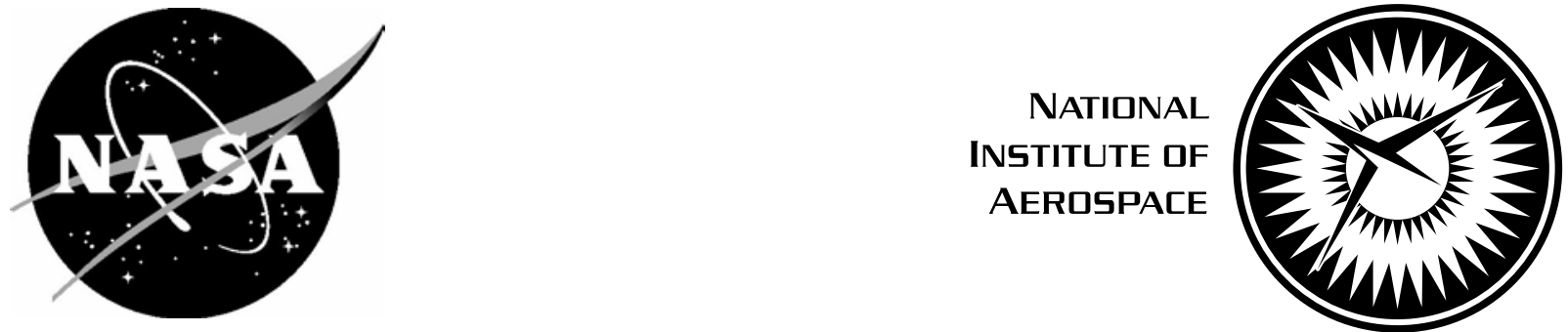

\title{
Gain-Scheduled Fault Tolerance Control Under False Identification
}

\author{
Jong-Yeob Shin
}

National Institute of Aerospace, Hampton, Virginia 
Since its founding, NASA has been dedicated to the advancement of aeronautics and space science. The NASA Scientific and Technical Information (STI) Program Office plays a key part in helping NASA maintain this important role.

The NASA STI Program Office is operated by Langley Research Center, the lead center for NASA's scientific and technical information. The NASA STI Program Office provides access to the NASA STI Database, the largest collection of aeronautical and space science STI in the world. The Program Office is also NASA's institutional mechanism for disseminating the results of its research and development activities. These results are published by NASA in the NASA STI Report Series, which includes the following report types:

- TECHNICAL PUBLICATION. Reports of completed research or a major significant phase of research that present the results of NASA programs and include extensive data or theoretical analysis. Includes compilations of significant scientific and technical data and information deemed to be of continuing reference value. NASA counterpart of peerreviewed formal professional papers, but having less stringent limitations on manuscript length and extent of graphic presentations.

- TECHNICAL MEMORANDUM. Scientific and technical findings that are preliminary or of specialized interest, e.g., quick release reports, working papers, and bibliographies that contain minimal annotation. Does not contain extensive analysis.

- CONTRACTOR REPORT. Scientific and technical findings by NASA-sponsored contractors and grantees.
- CONFERENCE PUBLICATION. Collected papers from scientific and technical conferences, symposia, seminars, or other meetings sponsored or co-sponsored by NASA.

- SPECIAL PUBLICATION. Scientific, technical, or historical information from NASA programs, projects, and missions, often concerned with subjects having substantial public interest.

- TECHNICAL TRANSLATION. Englishlanguage translations of foreign scientific and technical material pertinent to NASA's mission.

Specialized services that complement the STI Program Office's diverse offerings include creating custom thesauri, building customized databases, organizing and publishing research results ... even providing videos.

For more information about the NASA STI Program Office, see the following:

- Access the NASA STI Program Home Page at http://www.sti.nasa.gov

- E-mail your question via the Internet to help@sti.nasa.gov

- Fax your question to the NASA STI Help Desk at (301) 621-0134

- Phone the NASA STI Help Desk at (301) 621-0390

- Write to:

NASA STI Help Desk

NASA Center for AeroSpace Information

7121 Standard Drive

Hanover, MD 21076-1320 
NASA/CR-2006-213925

NIA Report No. 2005-07
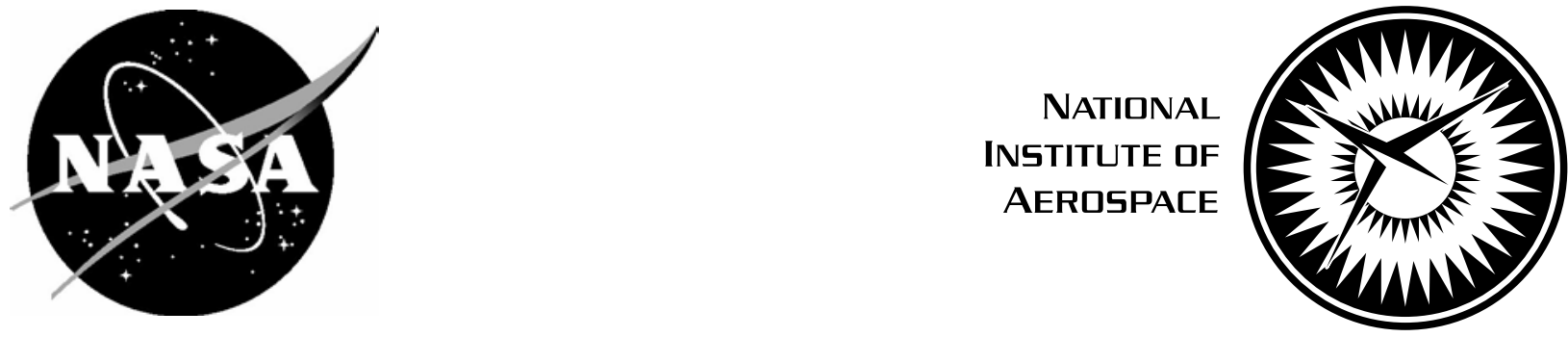

\section{Gain-Scheduled Fault Tolerance Control Under False Identification}

Jong-Yeob Shin

National Institute of Aerospace, Hampton, Virginia

National Aeronautics and

Space Administration 
Available from:

NASA Center for AeroSpace Information (CASI)

7121 Standard Drive

Hanover, MD 21076-1320

(301) 621-0390
National Technical Information Service (NTIS) 5285 Port Royal Road Springfield, VA 22161-2171

(703) 605-6000 


\title{
GAIN-SCHEDULED FAULT TOLERANCE CONTROL UNDER FALSE IDENTIFICATION*
}

\author{
Jong-Yeob Shin ${ }^{\dagger}$
}

\begin{abstract}
An active fault tolerant control (FTC) law is generally sensitive to false identification since the control gain is reconfigured for fault occurrence. In the conventional FTC law design procedure, dynamic variations due to false identification are not considered. In this paper, an FTC synthesis method is developed in order to consider possible variations of closed-loop dynamics caused by false identification. An active FTC synthesis problem is formulated into a linear matrix inequality (LMI) optimization problem to minimize the upper bound of the induced- $\mathcal{L}_{2}$ norm which can represent the worst-case performance degradation due to false identification. The developed synthesis method is applied to control of the longitudinal motion of FASER (Free-flying Airplane for Subscale Experimental Research). The designed FTC law of the airplane is simulated for pitch angle command tracking under a false identification case.
\end{abstract}

\section{INTRODUCTION}

In the past few decades, there has been interest in a fault tolerant control (FTC) system which has the ability to detect actuator/sensor faults automatically and to prevent faults from developing into a total system failure. Especially in constructing a flight control system, an active FTC system has been researched for achieving single aircraft accident prevention $[2,3,7,16]$. An active FTC system consists of an FTC law, a fault detection and isolation (FDI) module and a supervisory system. An FTC law should react to actuator/sensor faults through reconfiguration and an FDI module should detect actuator/sensor fault occurrences. Based on the information provided by an FDI module, a supervisory system decides which actuator/sensor is faulty and sends a signal to an FTC law for its reconfiguration.

In general, an active FTC law is designed, based on an open-loop system modeled as a function of fault parameters under the assumption that they are immediately identified by an FDI module. Recently, using linear matrix inequality (LMI) optimization solutions [3, 7, 16], an active FTC law has been synthesized in the form of a linear parameter varying (LPV) system whose dynamics vary as scheduling parameters change. Open-loop dynamics are modeled as an LPV system in which some of the scheduling parameters are fault parameters that represent fault occurrences at actuators/sensors. The designed LPV-FTC law can robustly stabilize the closed-loop system and achieve desired performance during a fault occurrence under the assumption that fault parameters are measured in real-time.

${ }^{*}$ This work was supported by the National Aeronautics and Space Administration under NASA Cooperative Agreement NCC1-02043.

${ }^{\dagger}$ National Institute of Aerospace (N.I.A.), Hampton, VA 23666. Email:shinjy@nianet.org 
Typically, there is always some level of time-delay to detect faults regardless of FDI algorithms such as an extended Kalman FDI filter [16] or an LPV-FDI filter [17]. During a time-delay interval, an open-loop system is in a faulty condition but the information provided by an FDI module implies that the system is not in a faulty condition. It is also possible that an FDI module and a supervisory system can produce false identification on healthy actuator/sensors, which may lead the system to be locally unstable temporarily.

The objectives of FTC synthesis are for the closed-loop system to be stable and to be able to achieve the desired performance level for fault occurrences and to reduce performance degradation due to false identification. One approach to achieve the objectives is designing a passive FTC law which has constant gains for both healthy and faulty systems. In that case, obviously the closed-loop system can be stable for a false identification case since the control gains are not changed due to false identification. The designed passive FTC law may be, however, conservative in performance since the controller is constructed from the common solutions of the LMI optimization problem[3] for both healthy and faulty systems.

Another approach is designing an active FTC law whose gains are reconfigured when a fault occurs. One of the synthesis methods to design an active FTC law is a conventional LPV synthesis method[18] in which it is assumed that scheduling parameters of an LPV system are exactly measured in real-time. In the conventional LPV synthesis method, false identification can not be considered. It is needed to modify the LPV synthesis method in order to consider possible closed-loop dynamic variations caused by false identification.

In this paper, an LPV control synthesis method is developed to design an active FTC law in order to consider the dynamic variations of an FTC system due to false identification, based on the brief unstable LPV system analysis method[13, 8] and the conventional LPV synthesis method[18]. In Ref.[8], the performance of an LPV system with brief instability was analyzed in terms of the upper bound of the $\mathcal{L}_{2}$ norm as a function of duration time and exponential decay rate over parameter subspaces. In Ref.[13], the LPV analysis method[8] is applied to analyze an FTC system under false identification. In Ref.[13], during a short time interval of false identification, the closed-loop system can be locally unstable. The unstable rate was calculated and then used to calculate the upper bound of the $\mathcal{L}_{2}$ performance level over all parameter spaces. In this paper, an LPV control synthesis method under possible false identification is developed and applied for designing an active FTC laws of the longitudinal motion of FASER.

This paper is organized as follows. In Section 2, the analysis problem including false identification case is described and in Section 3 a gain-scheduled FTC synthesis methodology is presented. In Section 4, the longitudinal motion of FASER is described in terms of control sensitivities of each control surfaces and the details in designing FTC law are presented. Section 5 concludes with a brief summary.

\section{LINEAR PARAMETER VARYING ANALYSIS METHOD}

An FTC system consists of an FTC law, an FDI module and a supervisory system (logic). When a fault occurs, the FDI module and supervisory system detect it and generate signals for evaluating/reconfiguring the FTC law. The FDI model requires some time to detect a fault. Hereafter, the required time is called detection time for the FDI module. During 
detection time, open-loop dynamics are in a faulty condition but the designed control law is not yet reconfigured for it. Suppose there are redundant control actuators to prevent a system having zero controllability for fault occurrence. The FDI module can also identify a healthy actuator as a faulty one for a short time interval when a fault occurs on another. It is called false identification. In Refs.[13, 8, 15], an analysis framework of the FTC system is developed for false identification and detection time-delay cases. In this section, the LPV analysis method including false identification is summarized.

Consider an open-loop system as an LPV system:

$$
\left[\begin{array}{l}
\dot{x} \\
e \\
y
\end{array}\right]=\left[\begin{array}{ccc}
A\left(\rho_{p}, \rho_{f}\right) & B_{1}\left(\rho_{p}, \rho_{f}\right) & B_{2}\left(\rho_{p}, \rho_{f}\right) \\
C_{1}\left(\rho_{p}, \rho_{f}\right) & D_{11}\left(\rho_{p}, \rho_{f}\right) & D_{12}\left(\rho_{p}, \rho_{f}\right) \\
C_{2}\left(\rho_{p}, \rho_{f}\right) & D_{21}\left(\rho_{p}, \rho_{f}\right) & 0
\end{array}\right]\left[\begin{array}{l}
x \\
d \\
u
\end{array}\right]
$$

where $\rho_{p} \in \mathcal{R}^{n_{p}}$ are physical parameters measurable by sensors in real-time such as velocity and angle of attack in flight dynamics and $\rho_{f} \in \mathcal{R}^{n_{f}}$ are fault parameters estimated by the FDI module that can indicate open-loop dynamic variations due to fault occurrence. The vectors $x \in \mathcal{R}^{n_{x}}, e \in \mathcal{R}^{n_{e}}, y \in \mathcal{R}^{n_{y}}, d \in \mathcal{R}^{n_{d}}$, and $u \in \mathcal{R}^{n_{u}}$ are states, errors, measurements, disturbances and input signals, respectively. Note that the matrices are functions of physical parameters and fault parameters.

A control law is defined as

$$
\left[\begin{array}{c}
\dot{x}_{k} \\
u
\end{array}\right]=\left[\begin{array}{cc}
A_{k}\left(\rho_{p}, \bar{\rho}_{f}\right) & B_{k}\left(\rho_{p}, \bar{\rho}_{f}\right) \\
C_{k}\left(\rho_{p}, \bar{\rho}_{f}\right) & 0
\end{array}\right]\left[\begin{array}{c}
x_{k} \\
y
\end{array}\right]:=K\left[\begin{array}{c}
x_{k} \\
y
\end{array}\right]
$$

where the vector $x_{k}$ is a control state vector and $\bar{\rho}_{f}$ are estimated fault parameters. Note that the control gain is a function of physical parameters and estimated fault parameters.

Using Eqs.(1) and (2), the closed-loop system is

$$
\left[\begin{array}{c}
\dot{x}_{c l} \\
e
\end{array}\right]=\left[\begin{array}{ll}
A_{c l}\left(\rho_{p}, \rho_{f}, \bar{\rho}_{f}\right) & B_{c l}\left(\rho_{p}, \rho_{f}, \bar{\rho}_{f}\right) \\
C_{c l}\left(\rho_{p}, \rho_{f}, \bar{\rho}_{f}\right) & D_{c l}\left(\rho_{p}, \rho_{f}, \bar{\rho}_{f}\right)
\end{array}\right]\left[\begin{array}{c}
x_{c l} \\
d
\end{array}\right]
$$

where $x_{c l}^{T}=\left[\begin{array}{ll}x^{T} & x_{k}^{T}\end{array}\right] \in \mathcal{R}^{n}$ and

$$
\begin{aligned}
A_{c l}(\rho) & =\tilde{A}\left(\rho_{p}, \rho_{f}\right)+\hat{B}\left(\rho_{p}, \rho_{f}\right) K\left(\rho_{p}, \bar{\rho}_{f}\right) \hat{C}\left(\rho_{p}, \rho_{f}\right) \\
B_{c l}(\rho) & =\tilde{B}\left(\rho_{p}, \rho_{f}\right)+\hat{B}\left(\rho_{p}, \rho_{f}\right) K\left(\rho_{p}, \bar{\rho}_{f}\right) \hat{D}_{21}\left(\rho_{p}, \rho_{f}\right) \\
C_{c l}(\rho) & =\tilde{C}\left(\rho_{p}, \rho_{f}\right)+\hat{D}_{12}\left(\rho_{p}, \rho_{f}\right) K\left(\rho_{p}, \bar{\rho}_{f}\right) \hat{C}\left(\rho_{p}, \rho_{f}\right) \\
D_{c l}(\rho) & =D_{11}\left(\rho_{p}, \rho_{f}\right)+\hat{D}_{12}\left(\rho_{p}, \rho_{f}\right) K\left(\rho_{p}, \bar{\rho}_{f}\right) \hat{D}_{21}\left(\rho_{p}, \rho_{f}\right)
\end{aligned}
$$

where the parameter matrices are

$$
\tilde{A}=\left[\begin{array}{cc}
A & 0 \\
0 & 0
\end{array}\right], \quad \tilde{B}=\left[\begin{array}{c}
B_{1} \\
0
\end{array}\right], \quad \tilde{C}=\left[\begin{array}{ll}
C_{1} & 0
\end{array}\right], \quad \hat{C}=\left[\begin{array}{cc}
0 & I \\
C_{2} & 0
\end{array}\right]
$$




$$
\hat{B}=\left[\begin{array}{cc}
0 & B_{2} \\
I & 0
\end{array}\right], \quad \hat{D}_{12}=\left[\begin{array}{ll}
0 & D_{12}
\end{array}\right], \quad \hat{D}_{21}=\left[\begin{array}{c}
0 \\
D_{21}
\end{array}\right]
$$

Hereafter, parameters $\left(\rho_{p}, \rho_{f}, \bar{\rho}_{f}\right)$ are denoted by $\rho$. Note that when an FDI module identifies faults immediately, the estimated fault parameters $\bar{\rho}_{f}$ are equal to $\rho_{f}$. For analyzing the closed-loop system for false identification, $\bar{\rho}_{f}$ and $\rho_{f}$ are treated as independent parameters to capture the closed-loop dynamic variations due to false identification.

The trajectories of parameters $\rho(t)$ stay in the compact set called the entire parameter space such that

$$
\mathcal{P}:=\left\{\rho(t) \mid \rho_{p}(t) \in \mathcal{F}_{p}, \quad \rho_{f}(t) \in \mathcal{F}_{\rho_{f}}, \quad \bar{\rho}_{f}(t) \in \mathcal{F}_{\bar{\rho}_{f}}\right\}
$$

where $\mathcal{F}_{p}, \mathcal{F}_{\rho_{f}}$, and $\mathcal{F}_{\bar{\rho}_{f}}$ are bounded compact sets in $\mathcal{R}^{n_{p}}, \mathcal{R}^{n_{f}}$, and $\mathcal{R}^{n_{f}}$, respectively. Note that $\mathcal{F}_{p}$ is called flight envelope when $\rho_{p}$ are velocity and angle of attack for longitudinal motion of an airplane.

The entire parameter set is divided into two subspaces $\mathcal{P}_{1}$ and $\mathcal{P}_{2}$ such that

$$
\begin{aligned}
& \mathcal{P}:=\mathcal{P}_{1} \cup \mathcal{P}_{2}, \\
& \emptyset=\mathcal{P}_{1} \cap \mathcal{P}_{2},
\end{aligned}
$$

The subspaces are defined based on the closed-loop dynamic changes due to false identification. Here, when all parameters stay in $\mathcal{P}_{1}$, the FDI module estimates fault parameters accurately to within a small error bound, $\delta_{\rho}$. Thus, the subspace $\mathcal{P}_{1}$ can be defined as:

$$
\mathcal{P}_{1}:=\left\{\rho_{f}(t) \mid \rho_{f}(t) \in \mathcal{S}\left(\bar{\rho}_{f}, \delta_{\rho}\right), \bar{\rho}_{f}(t) \in \mathcal{F}_{\bar{\rho}_{f}}, \quad \rho_{p} \in \mathcal{F}_{p}\right\}
$$

where

$$
\mathcal{S}\left(\bar{\rho}_{f}, \delta_{\rho}\right):=\left\{\rho_{f}(t) \mid\left\|\rho_{f}(t)-\bar{\rho}_{f}(t)\right\| \leq \delta_{\rho}, 0 \leq \delta_{\rho}\right\} .
$$

For false identification, $\rho_{f}(t)$ is not in the set $\mathcal{S}\left(\bar{\rho}_{f}, \delta_{\rho}\right)$ since $\rho_{f}(t)$ indicates a healthy condition but $\bar{\rho}_{f}(t)$ indicates a faulty condition. Parameter subspace $\mathcal{P}_{2}$ is defined for the false identification. Assume that the closed-loop system is locally unstable along the trajectories over the parameter subspace $\mathcal{P}_{2}$.

To represent dynamic variations of the closed-system in Eq. (3) over each parameter subspace, a duration time over each subspace is defined as follows [8]:

Definition 1 Duration time $T_{p_{i}}$ over each parameter subspace :

$$
\begin{gathered}
T_{p_{i}}\left(t_{o}, t\right)=T_{o_{i}}+\int_{t_{o}}^{t} \sigma_{i}(\rho(s)) d s, \quad \forall t>t_{o}>0, \\
\sigma_{i}(\rho(s))=\left\{\begin{array}{ll}
0, & \rho(s) \notin \mathcal{P}_{i}, \\
1, & \rho(s) \in \mathcal{P}_{i},
\end{array} \quad i \in\left[\begin{array}{ll}
1 & 2
\end{array}\right] .\right.
\end{gathered}
$$

A duration time is bounded as

$$
T_{o_{i}} \leq T_{p_{i}} \leq T_{o_{i}}+\alpha_{i}\left(t-t_{o}\right)
$$


where $0 \leq \alpha_{i} \leq 1$ and $0 \leq T_{o_{i}}$. The constant $\alpha_{i}$ represents a ratio of the duration time in the $i$-th subspace to the total time and $T_{o_{i}}$ is the duration time for a system to stay in the $i$-th subspace during the interval $\left[0, t_{o}\right]$. Note that $\sum_{i=1}^{2} \alpha_{i}=1$ and $\sum_{i=1}^{2} T_{o_{i}}=t_{o}$.

For the closed-loop system of Eq. (3), an induced- $\mathcal{L}_{2}$ norm is defined as:

$$
\sup _{\rho \in \mathcal{P}, d \in \mathcal{L}_{2},\|d\|_{2} \neq 0} \frac{\|e\|_{2}}{\|d\|_{2}} .
$$

The performance level of the closed-loop system with false identification is calculated in terms of the induced- $\mathcal{L}_{2}$ norm as follows:

Theorem 1: Consider the closed-loop system in Eq.(3). Suppose there exists a positive definite matrix $P(\rho) \in \mathcal{R}^{n_{x} \times n_{x}}$ such that

$$
\begin{aligned}
& {\left[\begin{array}{ccc}
A_{c l}^{T}(\rho) P(\rho)+P(\rho) A_{c l}(\rho)+\dot{P}(\rho)+\lambda_{1} P(\rho) & P(\rho) B_{c l}(\rho) & C_{c l}^{T}(\rho) \\
B_{c l}^{T}(\rho) P(\rho) & -\gamma I & D_{c l}^{T}(\rho) \\
C_{c l}(\rho) & D_{c l}(\rho) & -\gamma I
\end{array}\right]<0, \quad \rho \in \mathcal{P}_{1},} \\
& {\left[\begin{array}{ccc}
A_{c l}^{T}(\rho) P(\rho)+P(\rho) A_{c l}(\rho)+\dot{P}(\rho)-\kappa P(\rho) & P(\rho) B_{c l}(\rho) & C_{c l}^{T}(\rho) \\
B_{c l}^{T}(\rho) P(\rho) & -\gamma I & D_{c l}^{T}(\rho) \\
C_{c l}(\rho) & D_{c l}(\rho) & -\gamma I
\end{array}\right]<0, \quad \rho \in \mathcal{P}_{2} .}
\end{aligned}
$$

where $\lambda_{1}$ and $\kappa$ are positive scalar constants. The induced- $\mathcal{L}_{2}$ norm from $d$ to $e$ of the closed-loop system with given $T_{o_{2}}$ is no larger than $M_{\gamma}$ where

$$
\begin{aligned}
M_{\gamma} & =\gamma \sqrt{\frac{e^{\left(\lambda_{1}+\kappa\right) T_{o_{2}} \lambda_{1}}}{\lambda}}, \\
\lambda & =\lambda_{1}-\left(\lambda_{1}+\kappa\right) \alpha_{2} .
\end{aligned}
$$

Under the condition $\alpha_{2}<\alpha_{t}:=\frac{\lambda_{1}}{\left(\lambda_{1}+\kappa\right)}$, the constant $\lambda$ is positive.

Proof: See Ref.[15].

The constant $M_{\gamma}$ represents the upper bound of the worst-case performance level due to false identification. Thus, a synthesis problem for a controller $K$, as defined in Eq.(2), can be formulated into an optimization problem:

$$
\min _{K, \gamma>0, P>0} M_{\gamma} \text {, s.t. Eqs.(15)-(17). }
$$

with pre-defined $\lambda_{1}$ and $\kappa$ values. The optimization problem of Eq.(20) is converted into an LMI optimization problem in the next section. 


\section{GAIN-SCHEDULED FTC SYNTHESIS METHODOLOGY}

The projection lemma in Ref. [5] plays a key role in converting the analysis problem of Eqs.(15)-(17) into a control synthesis problem. Here, for completeness, the lemma [5] is described as follows:

Lemma 1: Given a symmetric matrix $\Psi \in \mathcal{R}^{n \times n}$ and two matrices $Q$ and $R$ of column dimension $n$, consider the problem of finding some matrix $\Theta$ of compatible dimensions such that

$$
\Psi+R^{T} \Theta^{T} Q+Q^{T} \Theta R<0
$$

Suppose the matrices $\mathcal{N}_{R}$ and $\mathcal{N}_{Q}$ are the null space of $R$ and $Q$, respectively. Then Eq.(21) is solvable for $\Theta$ if and only if

$$
\mathcal{N}_{R}^{T} \Psi \mathcal{N}_{R}<0, \text { and } \mathcal{N}_{Q}^{T} \Psi \mathcal{N}_{Q}<0
$$

Proof: See Ref.[5].

To synthesize a gain-scheduled FTC law under false identification, $\lambda_{1}$ and $\kappa$ values are defined by a control designer. The solvability condition of synthesizing a gain-scheduled control under false identification is described as follows:

Theorem 2 Given the open-loop system in Eq.(1), the entire parameter spaces are partitioned (see Eq.(8)). Suppose there exist positive matrices $X(\rho)$ and $Y(\rho)$ that satisfy the following conditions

$$
\begin{gathered}
\mathcal{N}_{X}^{T}\left[\begin{array}{ccc}
A(\rho)^{T} X(\rho)+X(\rho) A(\rho)+\dot{X}+\lambda_{1} X(\rho) & X(\rho) B_{1}(\rho) & C_{1}^{T}(\rho) \\
B_{1}^{T}(\rho) X(\rho) & -\gamma I & D_{11}^{T}(\rho) \\
C_{1}(\rho) & D_{11}(\rho) & -\gamma I
\end{array}\right] \mathcal{N}_{X}<0, \rho \in \mathcal{P}_{1} \\
\mathcal{N}_{Y}^{T}\left[\begin{array}{ccc}
Y(\rho) A(\rho)+Y(\rho) A^{T}(\rho)-\dot{Y}+\lambda_{1} Y(\rho) & Y(\rho) C_{1}^{T}(\rho) & B_{1}(\rho) \\
C_{1}(\rho) Y(\rho) & -\gamma I & D_{11}(\rho) \\
B_{1}^{T}(\rho) & D_{11}^{T}(\rho) & -\gamma I
\end{array}\right] \mathcal{N}_{Y}<0, \rho \in \mathcal{P}_{1}, \\
\mathcal{N}_{X}^{T}\left[\begin{array}{ccc}
A(\rho)^{T} X(\rho)+X(\rho) A(\rho)+\dot{X}-\kappa X(\rho) & X(\rho) B_{1}(\rho) & C_{1}^{T}(\rho) \\
B_{1}^{T}(\rho) X(\rho) & -\gamma I & D_{11}^{T}(\rho) \\
C_{1}(\rho) & D_{11}(\rho) & -\gamma I
\end{array}\right] \mathcal{N}_{X}<0, \rho \in \mathcal{P}_{2}, \\
\mathcal{N}_{Y}^{T}\left[\begin{array}{ccc}
Y(\rho) A(\rho)+Y(\rho) A^{T}(\rho)-\dot{Y}-\kappa Y(\rho) & Y(\rho) C_{1}^{T}(\rho) & B_{1}(\rho) \\
C_{1}(\rho) Y(\rho) & -\gamma I & D_{11}(\rho) \\
B_{1}^{T}(\rho) & D_{11}^{T}(\rho) & -\gamma I
\end{array}\right] \mathcal{N}_{Y}<0, \rho \in \mathcal{P}_{2}, \\
\quad\left[\begin{array}{cc}
X(\rho) & I \\
I & Y(\rho)
\end{array}\right]
\end{gathered}
$$


with given constants $\lambda_{1}$ and $\kappa$, where

$$
\begin{aligned}
& N_{X}=\operatorname{Null}\left(\left[\begin{array}{lll}
B_{2}^{T}(\rho) & D_{12}^{T}(\rho) & 0
\end{array}\right]\right) \\
& N_{Y}=\operatorname{Null}\left(\left[\begin{array}{lll}
C_{2}(\rho) & D_{21}(\rho) & 0
\end{array}\right]\right) .
\end{aligned}
$$

Then the induced- $\mathcal{L}_{2}$ norm of $d$ to e of the closed-loop system of Eq.(3) is bounded by $M_{\gamma}$ of Eq.(18).

Proof. : Define matrices as follows:

$$
\begin{aligned}
R & =\left[\begin{array}{lll}
\hat{C}(\rho) & \hat{D}_{21}(\rho) & 0
\end{array}\right] \\
Q & =\left[\begin{array}{lll}
\hat{B}^{T}(\rho) P(\rho) & 0 & \hat{D}_{12}^{T}(\rho)
\end{array}\right] \\
\Psi & =\left[\begin{array}{ccc}
\tilde{A}^{T}(\rho) P(\rho)+P(\rho) \tilde{A}(\rho)+\lambda_{1} P(\rho)+\dot{P} & P(\rho) \tilde{B}(\rho) & \tilde{C}^{T}(\rho) \\
\tilde{B}^{T}(\rho) P(\rho) & -\gamma I & D_{11}^{T}(\rho) \\
\tilde{C}(\rho) & D_{11}(\rho) & -\gamma I
\end{array}\right]
\end{aligned}
$$

Using Eqs. (30)-(32) and (2), the LMI condition of Eq.(15) is equal to

$$
\Psi+R^{T} K^{T} Q+Q^{T} K R<0 .
$$

Using Lemma 1, the LMI condition of Eq. (33) is decomposed into the following two LMI conditions:

$$
\begin{aligned}
\mathcal{N}_{R}^{T} \Psi \mathcal{N}_{R} & <0 \\
\mathcal{N}_{Q}^{T} \Psi \mathcal{N}_{Q} & <0 .
\end{aligned}
$$

The positive matrix $P$ is parameterized as follows:

$$
P(\rho)=\left[\begin{array}{cc}
X(\rho) & N(\rho) \\
N^{T}(\rho) & X_{1}(\rho)
\end{array}\right], \quad P^{-1}(\rho)=\left[\begin{array}{cc}
Y(\rho) & M(\rho) \\
M^{T}(\rho) & Y_{1}(\rho)
\end{array}\right] .
$$

From Eqs.(30)-(32) and (36), it is proved that the LMI condition of Eq.(34) is equal to Eq.(23).

Eq.(31) is rewritten as

$$
Q=\left[\begin{array}{lll}
\hat{B}^{T}(\rho) & 0 & \hat{D}_{12}^{T}(\rho)
\end{array}\right] S(\rho)
$$

where

$$
S(\rho):=\left[\begin{array}{ccc}
P(\rho) & 0 & 0 \\
0 & I & 0 \\
0 & 0 & I
\end{array}\right] .
$$

Using Eq.(37), the nullspace $\mathcal{N}_{Q}$ is rewritten as

$$
\mathcal{N}_{Q}=S^{-1} \mathcal{N}\left(\left[\hat{B}^{T}(\rho) \quad 00 \quad \hat{D}_{12}^{T}(\rho)\right] .\right.
$$

After some algebraic manipulation with Eqs.(30)-(32) and (39), the LMI condition of Eq.(35) is equal to Eq.(24). Using the above method, it is proved that the LMI in Eq.(16) is converted into the two LMI conditions of Eqs.(25) and (26). 
The control synthesis problem can be solved using the LMI Toolbox [6] such that

$$
\min _{X(\rho), Y(\rho)} \gamma
$$

In order to solve the optimization problem, the basis functions of $X(\rho)$ and $Y(\rho)$ are required.

$$
X(\rho):=\sum_{i=1}^{n_{s}} f_{i}(\rho) X_{i}, \quad Y(\rho):=\sum_{i=1}^{n_{s}} g_{i}(\rho) Y_{i}
$$

where functions $f_{i}(\rho): \mathcal{R}^{n_{s}} \rightarrow \mathcal{R}$ and $g_{i}(\rho): \mathcal{R}^{n_{s}} \rightarrow \mathcal{R}$ are bounded and the unknown matrices $X_{i}$ and $Y_{i}$ are symmetric, respectively. Note that it is still unknown how to choose optimal basis functions for predicted performance level of the closed-loop system. Under the assumption that $D_{12}$ and $D_{21}$ are full-column and full-row rank for the entire parameter space, respectively, the controller is constructed from the solutions $X(\rho)$ and $Y(\rho)$ in Eq.(40) as follows:[1]

$$
\begin{aligned}
& D_{k}=0 \\
& B_{k}=N^{-1} \hat{B}_{k} \\
& C_{k}=\hat{C}_{k} M^{-T} \\
& A_{k}=N^{-1}\left\{X \dot{Y}+N \dot{M}^{T}+\hat{A}_{k}-X A Y-\hat{B}_{k} C_{2} Y-X B_{2} \hat{C}_{k}\right\} M^{-T}
\end{aligned}
$$

where

$$
\begin{aligned}
& \hat{B}_{k}=\frac{1}{\gamma} C_{2}^{T}\left(D_{21} G_{1} D_{21}^{T}\right)^{-1}+\left(X B_{1}+\frac{1}{\gamma} C_{1}^{T} D_{11}\right)\left(D_{21}^{T} D_{21}\right)^{-1} D_{21}^{T} \\
& \hat{C}_{k}=-\frac{1}{\gamma}\left(D_{12}^{T} G_{2} D_{12}\right)^{-1} B_{2}^{T}-D_{12}^{T}\left(D_{12} D_{12}^{T}\right)^{-1}\left(C_{1} Y-\frac{1}{\gamma} D_{11} B_{1}^{T}\right) \\
& \hat{A}_{k}=A^{T}\left[X B_{1}+\hat{B}_{k} D_{21} \quad C_{1}^{T}\right]\left[\begin{array}{cc}
\gamma I & -D_{11}^{T} \\
-D_{11}^{T} & \gamma I
\end{array}\right]^{-1}\left[\begin{array}{c}
B_{1}^{T} \\
C_{1} Y+D_{12} \hat{C}_{k}
\end{array}\right] \\
& G_{1}=\left(\gamma^{2} I-D_{11}^{T} D_{11}\right)^{-1}, \quad G_{2}=\left(\gamma^{2} I-D_{11} D_{11}^{T}\right)^{-1} .
\end{aligned}
$$

Note that the matrices in Eqs.(42)-(48) are functions of the scheduling parameters. To complete the control construction, the following factorization is used

$$
N(\rho)=X(\rho), \quad M(\rho)=X(\rho)^{-1}-Y(\rho)
$$

to satisfy the equality condition:

$$
I-X(\rho) Y(\rho)=N(\rho) M^{T}(\rho) .
$$

\section{EXAMPLE}

In this section, it is demonstrated how to synthesize a gain-scheduled FTC law for the airplane of FASER shown in Fig. 1, by using the method described in the previous section. Two gain-scheduled LPV FTC laws are designed by the conventional LPV synthesis and the suggested method, respectively. For comparison, the designed control laws will be simulated under false identification. 


\subsection{Mathematical Model of FASER}

\subsubsection{Description of FASER}

FASER (Free-flying Airplane for Sub-scale Experimental Research) shown in Fig. 1 has been developed at the NASA Langley Research Center, which has a conventional high wing with tail configuration with $7 \mathrm{ft}$ wingspan and a propeller driven by an electric motor[11]. The detailed shape and physical quantities are taken from Refs.[10, 11]. The control surfaces of the airplane are an elevator, ailerons, flaps and a rudder driven by JRDS8611 Digital servos. For an INS system of the airplane, the MIDG GPS/INS unit built by Microbotics[9] is used. The positions are calculated based on GPS signals received by the MIDG. To measure angle of attack and side-slip angles, small props with small potentiometers are attached at both tips of the main wing. The roll, pitch and yaw angle rates are also measured by the MIDG. The on-board flight computer of FASER is the PC104 with $133 \mathrm{MHz} \mathrm{CPU}$, the 10 channel PWM board (Quartz-MM Diamond system) and the 16 channel analog I/O board (DM6420 Real-Time Devices).

\subsubsection{Nonlinear Equations of Longitudinal Motion}

The longitudinal dynamics have four states: velocity $(V)$ in $f t / s$, angle of attack $(\alpha)$ in radians, pitch rate $(q)$ in $\mathrm{rad} / \mathrm{s}$, and pitch angle $(\theta)$ in radians, and inputs: elevator deflection $\left(\delta_{e}\right)$ in radians and thrust $(T)$ in lbs. The airplane (FASER) has the right/left ailerons $\left(\delta_{r a} / \delta_{l a}\right)$ and the right/left flaps $\left(\delta_{r f} / \delta_{l f}\right)$ to be operated independently. The longitudinal equations of the motion are written as:

$$
\begin{aligned}
\dot{V} & =\frac{1}{m}\left\{\left(\bar{q} S C_{X}+T\right) \cos \alpha+\bar{q} S C_{Z} \sin \alpha\right\}+g(-\sin \theta \cos \alpha+\cos \theta \sin \alpha), \\
\dot{\alpha} & =q+\frac{\bar{q} S C_{Z} \cos \alpha}{m V}-\frac{\bar{q} S C_{X}+T}{m V}+\frac{g}{V}(\cos \alpha \cos \theta+\sin \theta \sin \alpha), \\
\dot{q} & =\frac{1}{J_{y y}} \bar{q} S \bar{c} C_{m}, \\
\dot{\theta} & =q,
\end{aligned}
$$

where aerodynamics coefficients $C_{X}, C_{Z}$, and $C_{m}$ are written as

$$
\begin{aligned}
C_{X} & =C_{X_{o}}(\alpha)+C_{X_{q}}(\alpha) \frac{\bar{c} q}{2 V}+C_{X_{\delta_{e}}}(\alpha) \delta_{e}+C_{X_{\delta_{a}}}(\alpha) \delta_{a_{a l l}}+C_{X_{\delta_{f}}}(\alpha) \delta_{f_{\text {all }}}, \\
C_{Z} & =C_{Z_{o}}(\alpha)+C_{Z_{q}}(\alpha) \frac{\bar{c} q}{2 V}+C_{Z_{\delta_{e}}}(\alpha) \delta_{e}+C_{Z_{\delta_{a}}}(\alpha) \delta_{a_{a l l}}+C_{Z_{\delta_{f}}}(\alpha) \delta_{f_{a l l}}, \\
C_{m} & =C_{m_{o}}(\alpha)+C_{m_{q}}(\alpha) \frac{\bar{c} q}{2 V}+C_{m_{\delta_{e}}}(\alpha) \delta_{e}+C_{m_{\delta_{a}}}(\alpha) \delta_{a_{a l l}}+C_{m_{\delta_{f}}}(\alpha) \delta_{f_{\text {all }}}
\end{aligned}
$$

Note that the positive deflection of both right and left aileron control surfaces is defined in the same way of the conventional definition of the positive elevator deflection. In the longitudinal motion, assume that the left and right aileron surfaces can symmetrically move 
to change the lift and drag forces. Thus, the control inputs of the airplane in the longitudinal motion are thrust $T(l b)$, elevator deflection $\delta_{e}(\mathrm{deg})$, aileron deflection $\delta_{a_{a l l}}=0.5\left(\delta_{r a}+\delta_{l a}\right)$, and flap deflection $\delta_{f_{a l l}}=0.5\left(\delta_{r f}+\delta_{l f}\right)$. The detailed aerodynamic coefficients are calculated by using a CFD code in Refs. $[4,12]$.

\subsubsection{Quasi-LPV Model of Longitudinal Motion}

It is required to represent the nonlinear dynamics as a quasi-LPV model in order to design an LPV control law using the method described in Section 3. Using the quasi-LPV representation method in Ref.[14], the nonlinear equation of longitudinal motion can be represented as the quasi-LPV model:

$$
\dot{x}=A(V, \alpha) x+B(V, \alpha) u
$$

where $x=\left[\begin{array}{llll}V & \alpha & q & \theta\end{array}\right]^{T}$ and $u=\left[\begin{array}{lll}T & \delta_{e} & \delta_{a_{a l l}}\end{array}\right]^{T}$. The detailed matrices $A$ and $B$ can be found in Refs. $[14,12]$. Note that the flaps are not included in the quasi-LPV model on purpose since the wake generated by flap movement may be able to coupled with elevator deflection movement. The coupling effect may generate mathematically unpredictable aerodynamic force and moment on the airplane. Hereafter, control variables are set as thrust $T$, elevator deflection $\delta_{e}$ and same directional aileron deflection $\delta_{a_{a l l}}$.

The flight envelope is defined as follows:

$$
\begin{aligned}
60 \mathrm{ft} / \mathrm{sec} & \leq V \leq 120 \mathrm{ft} / \mathrm{sec} \\
2^{\circ} & \leq \alpha \leq 7^{\circ}
\end{aligned}
$$

based on the trim region (the line of the plot shown in Fig.2) for a constant level flight condition $(\dot{x}=0, \alpha=\theta)$. Note that the states $V$ and $\alpha$ are also considered as scheduling parameters $\rho_{p}$ which represent flight conditions. The control action limits under the nominal condition are as follows:

$$
0 \leq T \leq 6 \mathrm{lb}, \quad-20^{\circ} \leq \delta_{e} \leq 20^{\circ}, \quad-20^{\circ} \leq \delta_{a_{a l l}} \leq 20^{\circ}
$$

\subsubsection{Elevator Fault Model and Parameter Space}

In this example, elevator failure is considered as loss of elevator controllability. Assume that the elevator is fixed at trim position for a failure case. Floating control surfaces, partially damaged control surfaces and control surfaces fixed at any other positions can be considered as failures and can be modeled. However, those failures are not considered here for simplicity. In this paper, the fault is simply modeled as linear change of the matrix $B(\rho)$ such as

$$
B(\rho) u=B\left(\rho_{p}\right)\left[\begin{array}{ccc}
1 & 0 & 0 \\
0 & \tau_{1} & 0 \\
0 & 0 & \tau_{2}
\end{array}\right] u
$$


where the failure parameters $\tau_{1}$ and $\tau_{2}$ can vary from 0 (failure condition) to 1 (healthy condition).

Consider that one of two actuators can be failed at a time in order to prevent zero controllability of the system. In order to represent the failure condition, the fault parameter $\rho_{f}$ is defined as follows:

$$
\rho_{f}(t)= \begin{cases}\tau_{1}(t) & \text { if } 0 \leq \tau_{1}(t)<1, \quad \tau_{2}=1 \\ 2-\tau_{2}(t) & \text { if } \tau_{1}=1, \quad 0 \leq \tau_{2}<1\end{cases}
$$

The estimated fault parameter can be calculated from the estimations of the failure parameters $\tau_{1}$ and $\tau_{2}$.

The false identification case is considered as the estimated fault parameter indicates the aileron failure for the elevator failure for a short time interval. In the time interval, aileron actuator is actually healthy but an FDI module indicates it as a faulty one. Also, the elevator actuator is failed but a FDI indicates it as a healthy one. In that case, estimated fault parameter $\bar{\rho}_{f}$ is equal to 2 (aileron failure) instead of 0 (elevator failure).

The entire parameter space $\mathcal{P}$ and subspaces shown in Fig. 3 are defined as

$$
\mathcal{P}=\mathcal{P}_{1} \cup \mathcal{P}_{2}
$$

where

$$
\begin{aligned}
& \mathcal{P}_{1}:=\left\{\rho \mid \rho_{p} \in \mathcal{F}_{p}, \quad \rho_{f} \in \mathcal{S}\left(\bar{\rho}_{f}, 0.1\right), \bar{\rho}_{f} \in\left[\begin{array}{ll}
0 & 2
\end{array}\right]\right\} \\
& \mathcal{P}_{2}:=\left\{\rho \mid \rho_{f}=0, \bar{\rho}_{f} \in\left[\begin{array}{ll}
1.9 & 2
\end{array}\right]\right\} .
\end{aligned}
$$

Here, the set $\mathcal{S}\left(\bar{\rho}_{f}, 0.1\right)$ is defined as

$$
\mathcal{S}\left(\bar{\rho}_{f}, 0.1\right):=\left\{\rho_{f}(t) \mid\left\|\rho_{f}(t)-\bar{\rho}_{f}(t)\right\|<0.1\right\} .
$$

The set $\mathcal{F}_{p}$ is defined as the flight envelope in Eq.(56). Note that the fault parameter $\rho_{f}$ can vary abruptly from 1 (healthy condition) to 0 (faulty condition) but the estimated fault parameter can smoothly vary from 1 to 0 , based on the FDI module algorithm. Generally, the LPV-FTC law is designed for the subspace $\mathcal{P}_{1}$ in Refs. $[16,7]$ under the assumption that the FDI can estimate the fault parameter fast and accurately. In this paper, we consider cases where the closed-loop system stays in the parameter subspace $\mathcal{P}_{2}$ for a short time interval.

\subsection{Control Synthesis Framework}

The control design objectives are to track the pitch angle command with less than $4 \%$ tracking error under a nominal condition and to reduce tracking error under a faulty condition. In the control design procedure, the variations of the predicted closed-loop dynamics due to false identification are also considered by including LMI conditions of Eqs. (25) and (26) with the pre-defined parameter $\kappa$. Recall that the parameter $\kappa$ represents possible instability of the closed-loop system under false identification [13].

The control design problem is formulated as a model matching problem shown in Fig.4. 
In the block diagram shown in Fig.4, the block "Act" implies the actuator models. In this example, the mathematical models of the JRDS8611 servo motor and the electric motor are approximated as the first order filters $\frac{20}{s+20}$ and $\frac{5}{s+5}$, respectively. The first order model of the servo motor is approximated with constant one in the control design procedure to reduce the order of the designed control law. The first order model is, however, used for simulations of the closed-loop system. The block $W_{\text {noise }}$ represents sensor noise such as $\operatorname{diag}\left(\left[0.1 \mathrm{ft} / \mathrm{sec}, 1^{\circ} 1^{\circ}, 1\right]\right)$. The performance weighting function $W_{p}$ is set as $\frac{25}{s / 0.1+1}$ to represent $4 \%$ error allowance in a low frequency domain and the ideal transfer function $T_{i}$ is set as $\frac{1}{s / 0.9+1}$ for ideal pitch angle responses. The block $W_{\text {act }}$ is set as $\operatorname{diag}([1 / 10,1 / 401 / 40])$ to constrain control action. Using the block diagram shown in Fig.4, the augmented openloop is constructed with a linearized model around a trim point $\left(V_{t}, \alpha\right)=\left(70,5.4^{\circ}\right)$.

For simplicity in designing an FTC law under the false identification, the scheduling parameter is defined as the fault parameter $\rho_{f} \in[0,2]$ at the given trim point. The basis function $f_{i}(\rho)$ of $X$ in Eq. (41) is defined as

$$
f_{1}\left(\rho_{f}\right)=1, \quad f_{2}\left(\rho_{f}\right)=\rho_{f}, \quad f_{3}\left(\rho_{f}\right)=1 / \rho_{f},
$$

and the basis function $g_{i}(\rho)$ of $Y$ is set as same as $f_{i}(\rho)$. The LMI constraints of Eqs. (23)(26) are evaluated at each grid point in the set $\left[\begin{array}{llllll}0.01, & 0.2 & 0.4 & \cdots & 1.8 & 2\end{array}\right]$ of $\rho_{f}$. Note that the grid point of 0.01 that represents a faulty condition is chosen in order to avoid singularity of $1 / \rho_{f}$ when the basis functions are evaluated at every grid point. For evaluating the time derivatives of $X$ and $Y$, the parameter rate bound is defined as $1 \mathrm{sec}^{-1}$.

To solve the LMI optimization in Eqs.(23)-(26), the scalar parameters $\lambda_{1}$ and $\kappa$ are predefined as 0.01 and 0.1 , respectively. To calculate the best values of $\lambda_{1}$ and $\kappa$, it is required to solve another optimization by minimizing $M_{\gamma}$ of Eq. (18). In this paper, we choose them based on a few iterations of different values of $\lambda_{1}$ and $\kappa$. For example, when $\lambda_{1}$ is 0.2 , there is no feasible solution for the LMI optimization in Eqs. (23)-(26). Thus, $\lambda_{1}$ value should be smaller than 0.2 . When $\kappa$ is set as large number, it implies that the closed-loop system can be unstable with very fast rate. It also implies that the upper bound of the induced- $\mathcal{L}_{2}$ norm of Eq. (18) could be large. Thus, the parameter $\kappa$ can not be set as large number. When the parameter is set as small number close to zero, it implies that stability of the closedloop system is required under the false identification with expense of over all performance degradation.

In this paper, two LPV FTC controllers $K_{1}$ and $K_{2}$ are designed by the conventional LPV method and the method suggested in Section 3, respectively. The conventional LPV method can be re-constructed from Eqs. (23) and (24) by setting $\lambda_{1}$ as zero. The designed two LPV controllers are simulated in the next section.

\subsection{Simulation Results}

For all simulations, pitch angle commands are set as a square signal such as 1 degree up at 5 second and 1 degree down at 20 second. The label "Ti" in Figs. 5-9 denotes the ideal pitch angle time responses. The label $\delta_{e}$ and $\delta_{e_{c}}$ denote elevator deflection angle and elevator deflection angle command, respectively. For all simulations, state's initial values are set as the trim values. The simulation results under a healthy condition are shown in Fig. 5. Note 
that the controllers $K_{1}$ and $K_{2}$ achieve the desired performance objective that pitch angle command tracking is less than $4 \%$ at low frequency.

For an elevator failure case, the closed-loop time responses are shown in Fig. 6. In the simulation, it is assumed that elevator fails after 5 second and an FDI module detects the fault immediately. The pitch angle time responses with the controllers are very similar to each other and imply that the two controllers achieve the performance objective while the elevator fails. The two controllers generates aileron deflection angle commands to compensate the elevator failure.

Three false identification cases are simulated. 1) While the elevator fails after 5 second, an FDI detects the aileron failure between 5 and 10 seconds and the elevator failure after 10 second. 2) While the two actuators are healthy, an FDI detects elevator failure between 5 and 10 second. 3) While the two actuators are healthy, an FDI detects aileron failure between 5 and 10 second. The simulation results for the case 1 are shown in Fig. 7. Note that estimated fault parameter responses are generally simulated by an FDI module with the closed-loop system but in this paper, they are pre-defined in order to represent false identification. It is observed from Fig. 7 that the controller $K_{2}$ can reduce the performance degradation due to the false identification, but the controller $K_{1}$ can not. The controller $K_{1}$ generates elevator command signals due to the estimated fault parameter which indicates an aileron fault between $5 \mathrm{sec}$ and $10 \mathrm{sec}$. The generated elevator command signals can not operate the elevator actuator since the elevator is actually failed after 5 second. Again, in the design process for the controller $K_{1}$, there is assumption that the estimated fault parameter is equal to the true fault parameter. In the false identification, the assumption is violated. Meanwhile, in the controller $K_{2}$ design process, the possible false identification is considered. That leads to the reduction of performance degradation due to false identification. When the pre-defined parameters $\lambda_{1}$ and $\kappa$ are different values, the time responses of the closedloop system would be different. It would be of interest to further investigate the sensitivity the closed-loop performance and actuator responses to $\lambda_{1}$ and $\kappa$ for a false identification case. For the false identification cases 2 and 3, the closed-loop time responses are shown in Figs. 8 and 9, respectively. Note that the two controllers $K_{1}$ and $K_{2}$ can achieve the performance objective. Since the two actuators are healthy, one of compensated actuator command signals can operate healthy actuators, respectively. The second and third false identifications do not affect pitch angle tracking of the system, respectively.

\section{CONCLUSION}

In this paper, an active fault tolerant control synthesis method is developed under false identification. In the control synthesis method, possible closed-loop dynamic variations due to false identification are considered. In the control synthesis, the closed-loop system under false identification can be unstable for a brief time interval with trade-off of performance degradation. The performance level of the closed-loop system is described as a function of duration time of the parameter subspace, the induced $-\mathcal{L}_{2}$ norm index $\gamma$ and exponential decay rate in each parameter subspace. With given possible exponential decay rate of the predicted closed-loop system, the control synthesis problem is formulated into the LMI optimization to minimize the upper bound of the induced- $\mathcal{L}_{2}$ norm. The suggested LPV-FTC synthesis 
method is applied to design an FTC law for the longitudinal motion of the airplane FASER. According to simulation results, the designed control law is insensitive for false identification while it achieve pitch angle tracking error less than $4 \%$ in a faulty condition.

\section{ACKNOWLEDGMENTS}

The author thanks the technical monitor Dr. Irene Gregory at the NASA Langley Research Center and also acknowledge the contributions of David Cox (NASA Langley Research Center), and Pawel Chwalowski (Analytic Mechanics Associates) for developing the nonlinear mathematical model of the airplane.

\section{REFERENCES}

[1] Apkarian, P. and Adams, R. Advanced Gain-Scheduling Techniques for Uncertain Systems. IEEE Transactions on Control Systems Technology, 6(1):21-32, 1998.

[2] Belcastro, Christine and Belcastro, Celeste. Application of failure detection, identification, and accommodation methods for improved aircraft safety. In Proceedings of American Control Conference, pages 2623-2624. American Automatic Control Council, Evanston, IL, 2001.

[3] Chen, J., Patton, R.J., and Chen, Z. An LMI approach to Fault-Tolerant Control of Uncertain System. In Proceeding of the 1998 International Symposium on Intelligent Control, pages 175-180. Institute of Electrical and Electronics Engineers, Piscataway, N.J., 1998.

[4] Chwalowski, P. Vehicle Configuration Studies and Small Aircraft Formation Flying Study. Technical Report 04-33, Analytical Mechanics Associates, Inc., Nov. 2004.

[5] Gahinet, P. and Apkarian, P. A Linear Matrix Inequality Approach to $H_{\infty}$ Control. International Journal of Robust and Nonlinear Control, pages 421-448, 1994.

[6] Gahinet, P., Nemirovski, A., Laub, A.J., and Chilai. M. LMI Control Toolbox User's Guide. The MATH Works, Natick, Mass, 1995.

[7] Ganguli, S., Marcos, A., and Balas, G. Reconfigurable LPV Control Design for Boeing 747-100/200 Longitudinal Axis. In Proceedings of American Control Conference, pages 3612-3617, Anchorage, AK, 2002. American Control Conference Council, Evanston, IL.

[8] Hespanha, J., Yakimenko, O., Kaminer, I., and Pascoal, A. LPV Systems with Brief Instabilities:Application to Integrated Vision/IMU Navigation. In IEEE Proceeding of the Conference on Decision and Control, 2001.

[9] Microbotics, Inc. http://www.microboticsinc.com/midg.html. Microbotics, Inc., 2005.

[10] Monzon, B.R. Development of a Nonlinear Simulation for a Research Model Airplane. Master's thesis, MSE Thesis, Geroge Washington University JIAFS,, NASA Langley Research Center, September 2001. 
[11] Morelli, E.A. and DeLoach, R. Wind Tunnel Database Developement Using Mordern Experiment Design and Multivariate Orthogonal Functions. In 41st AIAA Aerospace Sciences Meeting and Exhibit, AIAA 2003-0653, Jan. 6-9 2003.

[12] Shin, J.-Y. Quasi-Linear Parameter Varying Representation of General Aircraft Dynamics Over Non-Trim Region. Technical report, National Institute of Aerospace, 2005.

[13] Shin, J-Y. and Belcastro, C. Analysis of A Fault Tolerant Control System: False Fault Detection Case. In 5th IFAC Symposium on Fault Detection, Supervision and Safety of Technical Processes, June 2003.

[14] Shin, J-Y. and Belcastro, C. Quasi-Linear Parameter Varying Representation over NonTrim Region. In AIAA Guidance, Navigation, and Control Conference and Exhibit, AIAA-2004-5423. American Institute of Aeronautics and Astronautics, 2004.

[15] Shin, J-Y., Wu, N.E., and Belcastro, C. Linear Parameter Varying Control Synthesis for Actuator Failure, Based on Estimated Parameter. In AIAA Guidance, Navigation and Control, number AIAA-2002-4546. American Institute of Aeronautics and Astronautics, 2002.

[16] Shin, J-Y., Wu, N.E., and Belcastro, C. Adaptive Linear Parameter Varying Control Synthesis for Actuator Failure. Journal of Guidance, Control, and Dynamics, 27(5):787794, Sept. 2004.

[17] Szaszi, I., Marcos, A., Balas, G., and Bokor, J. LPV Detection Filter Design for Boeing 747-100/200. In AIAA Guidance, Navigation and Control, number AIAA 2002-4957. American Institute of Aeronautics and Astronautics, 2002.

[18] Wu, F. Control of Linear Parameter Varying Systems. PhD thesis, Department of Mechanical Engineering, University of California, Berkeley, 1995. 


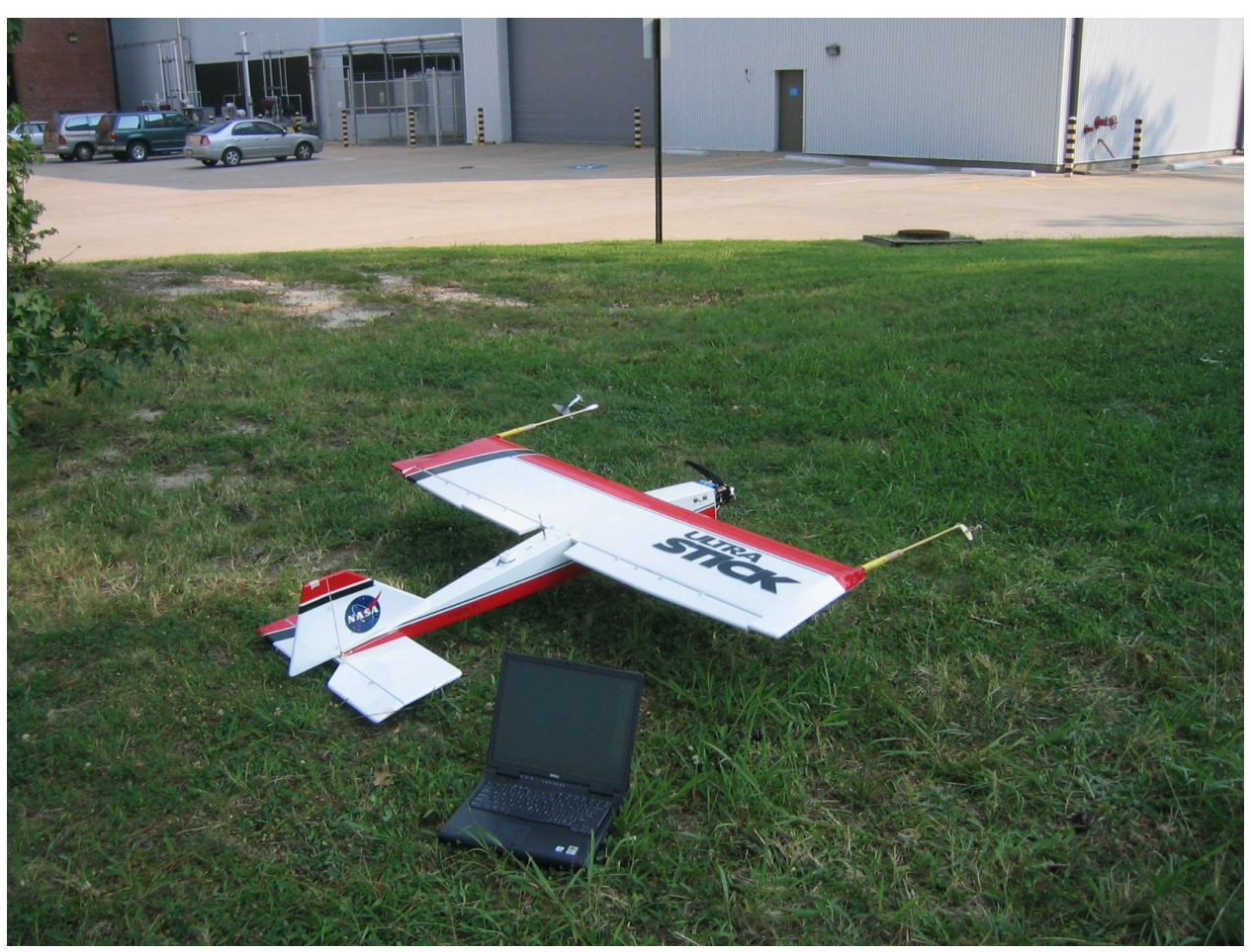

Figure 1: The FASER 


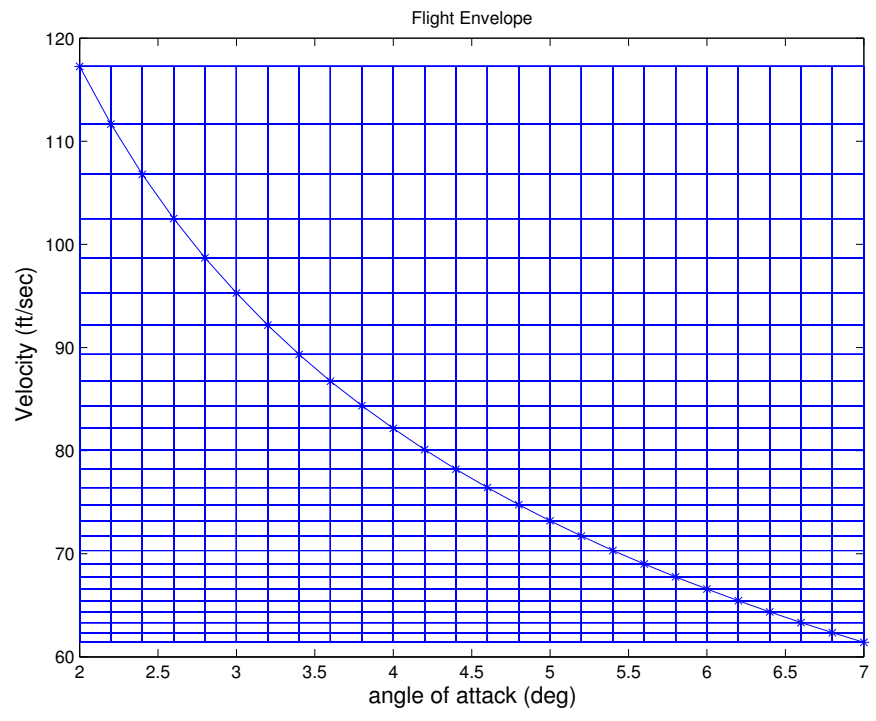

Figure 2: Flight envelop with trim points

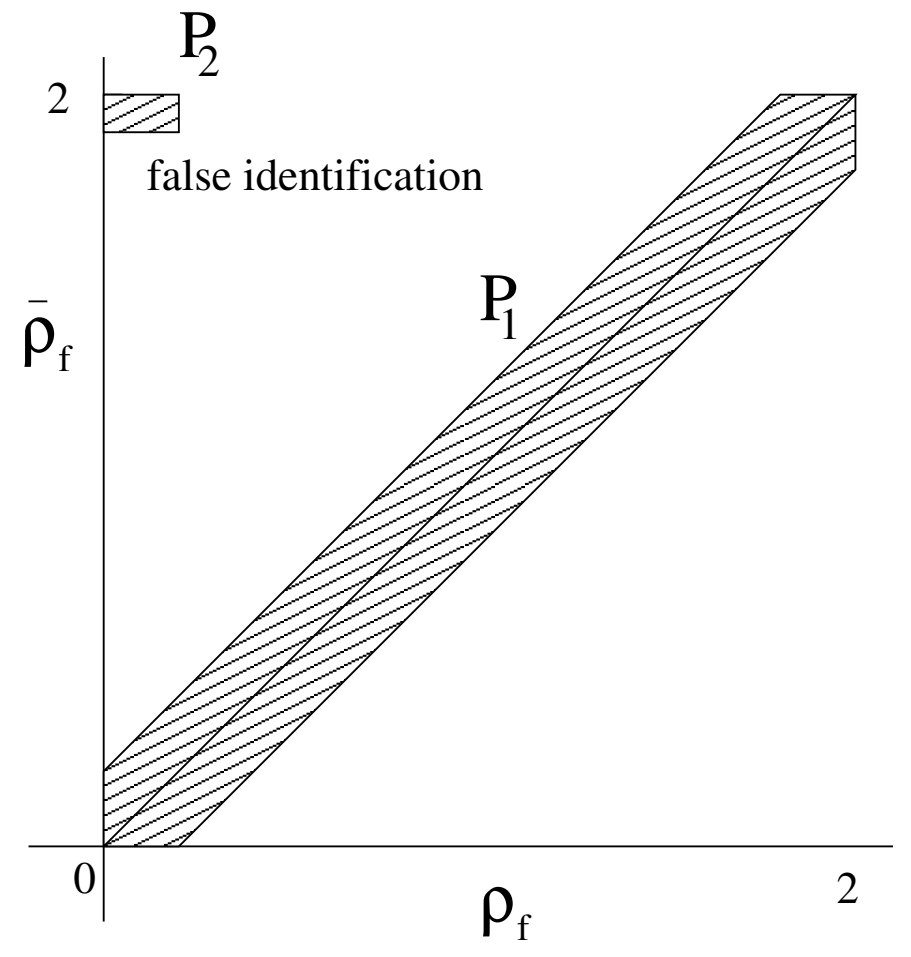

Figure 3: Parameter subspaces. 


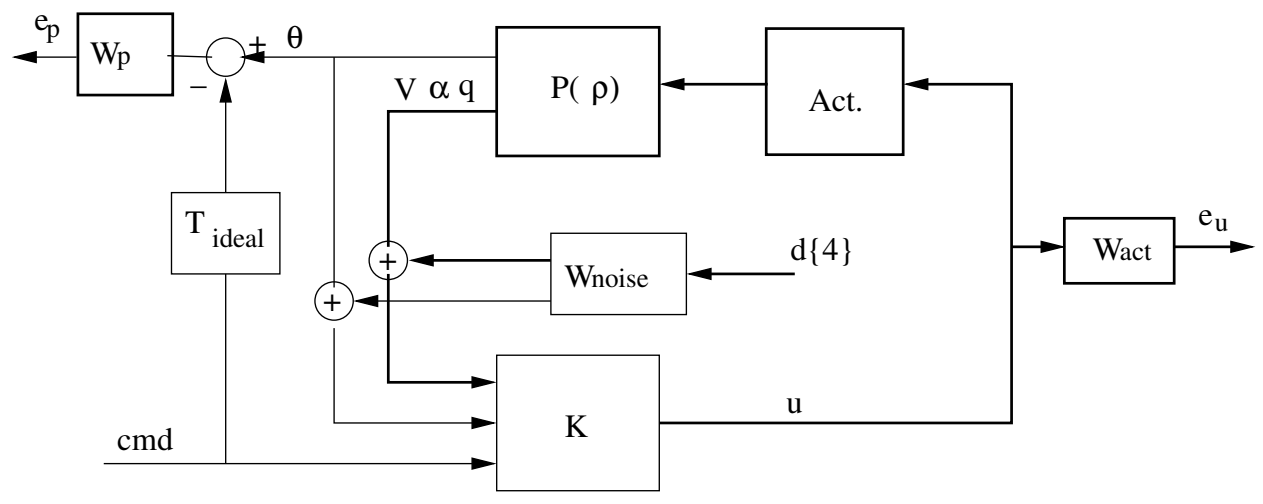

Figure 4: Model matching synthesis block diagram.
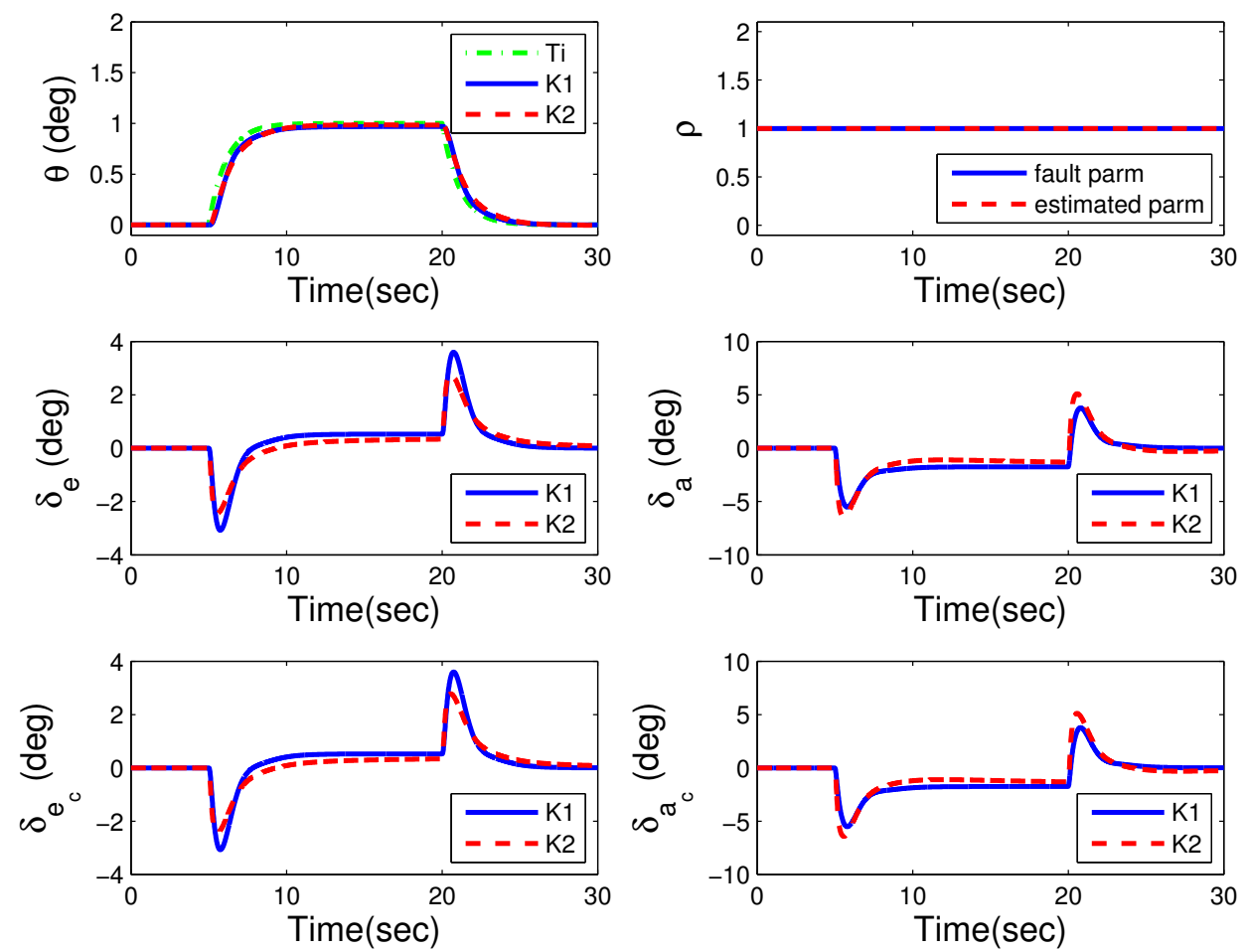

Figure 5: Time responses of the closed-loop system under a healthy condition. 

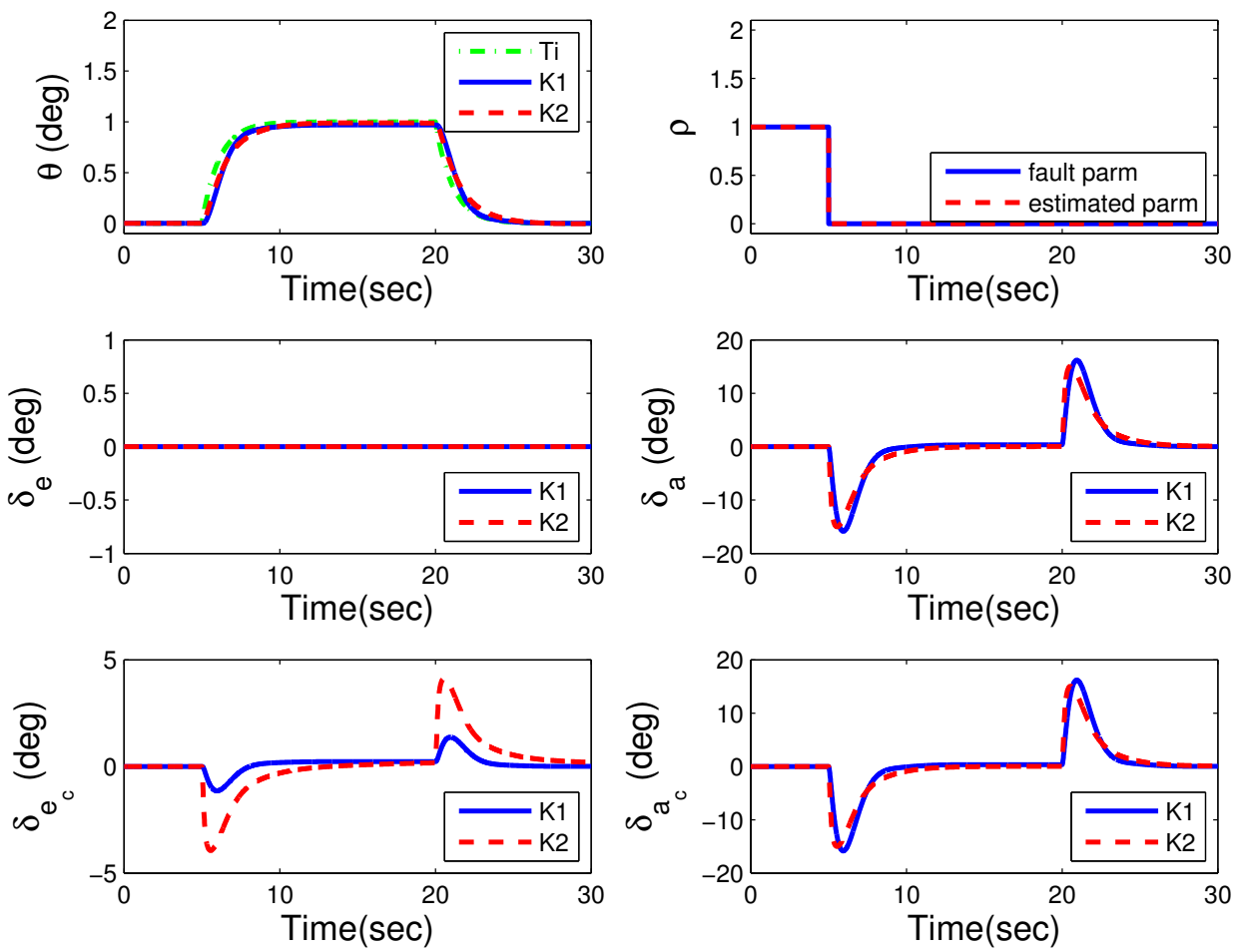

Figure 6: Time responses of the closed-loop system under elevator failure.
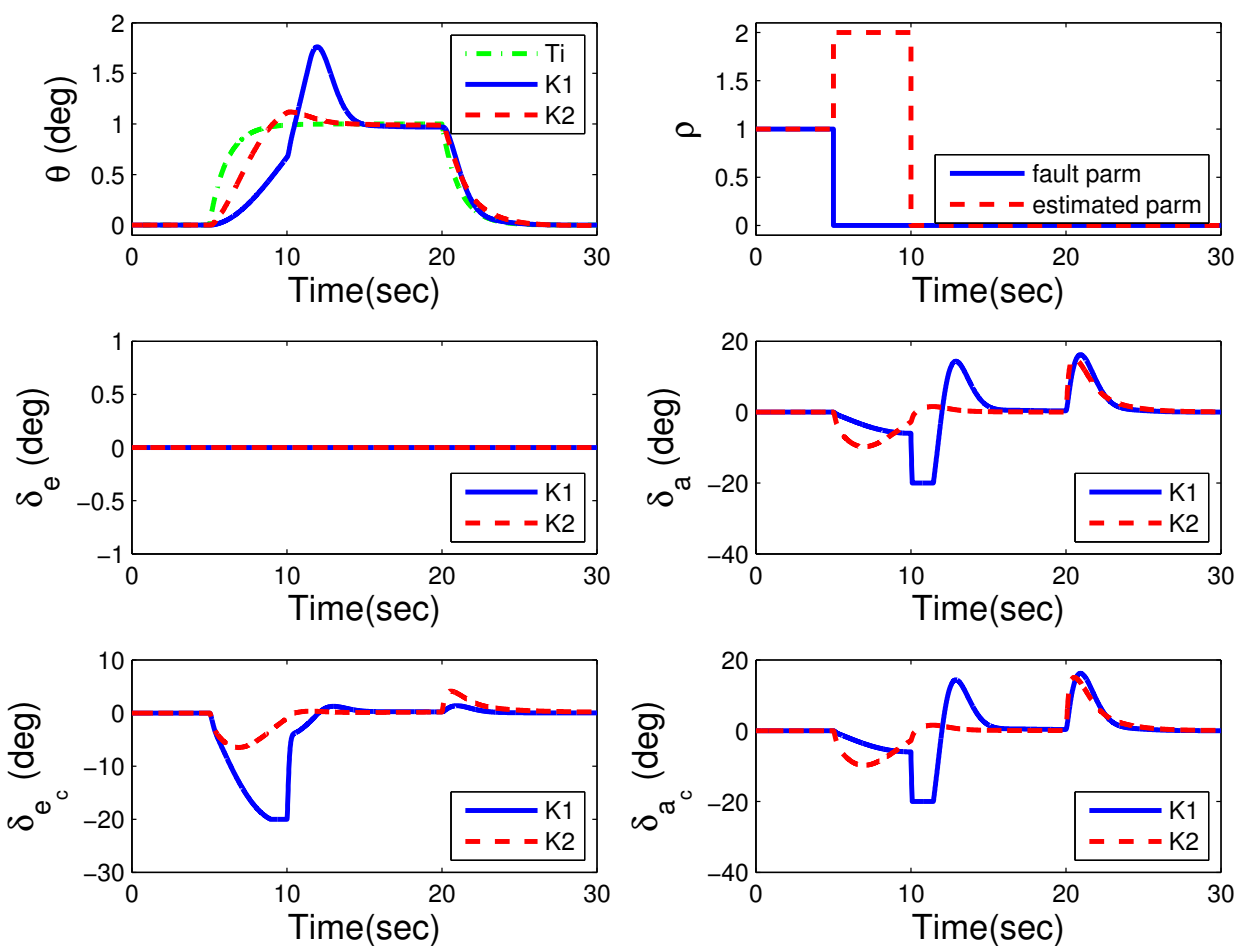

Figure 7: Time responses of the closed-loop system under the false identification case 1. 

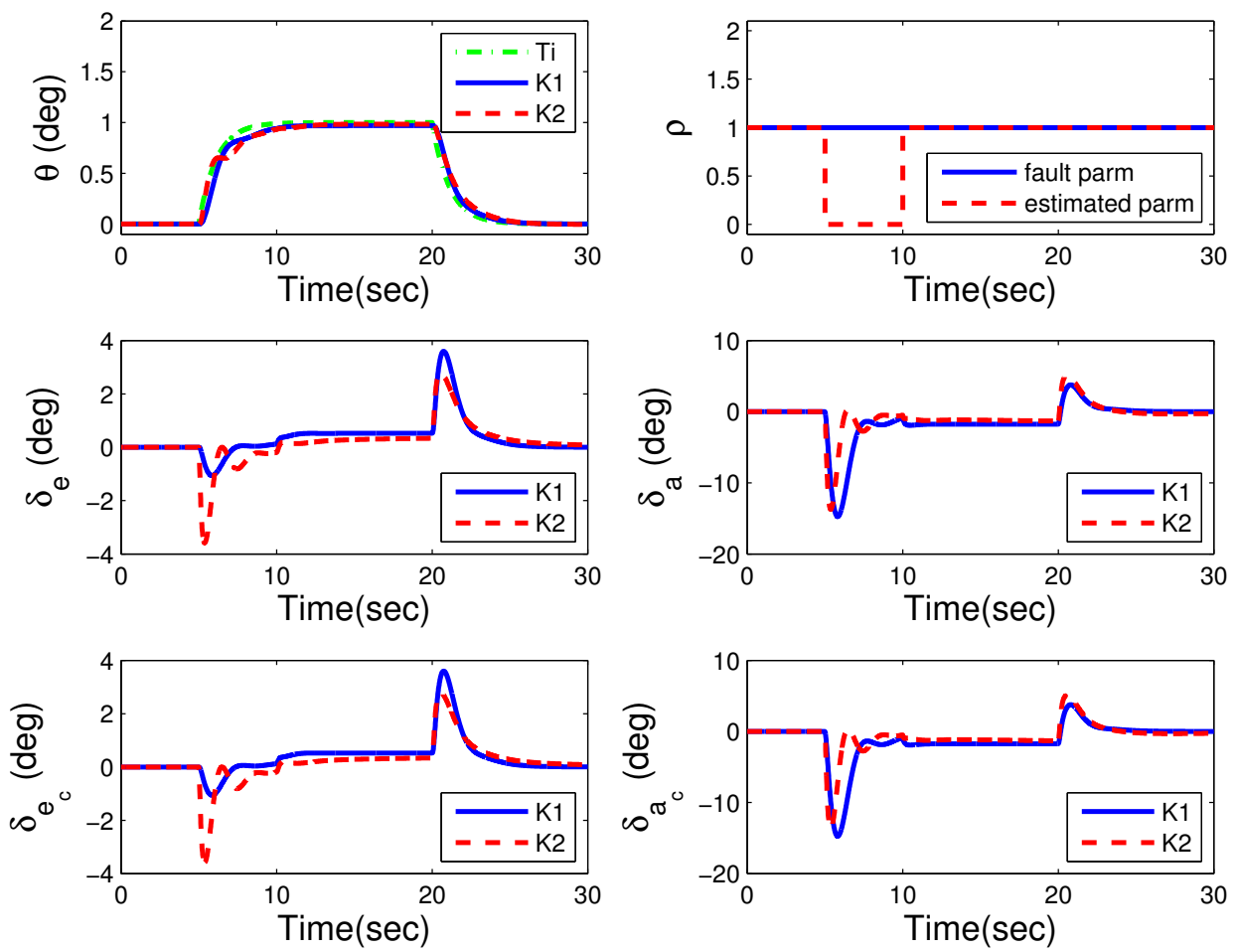

Figure 8: Time responses of the closed-loop system under the false identification case 2.
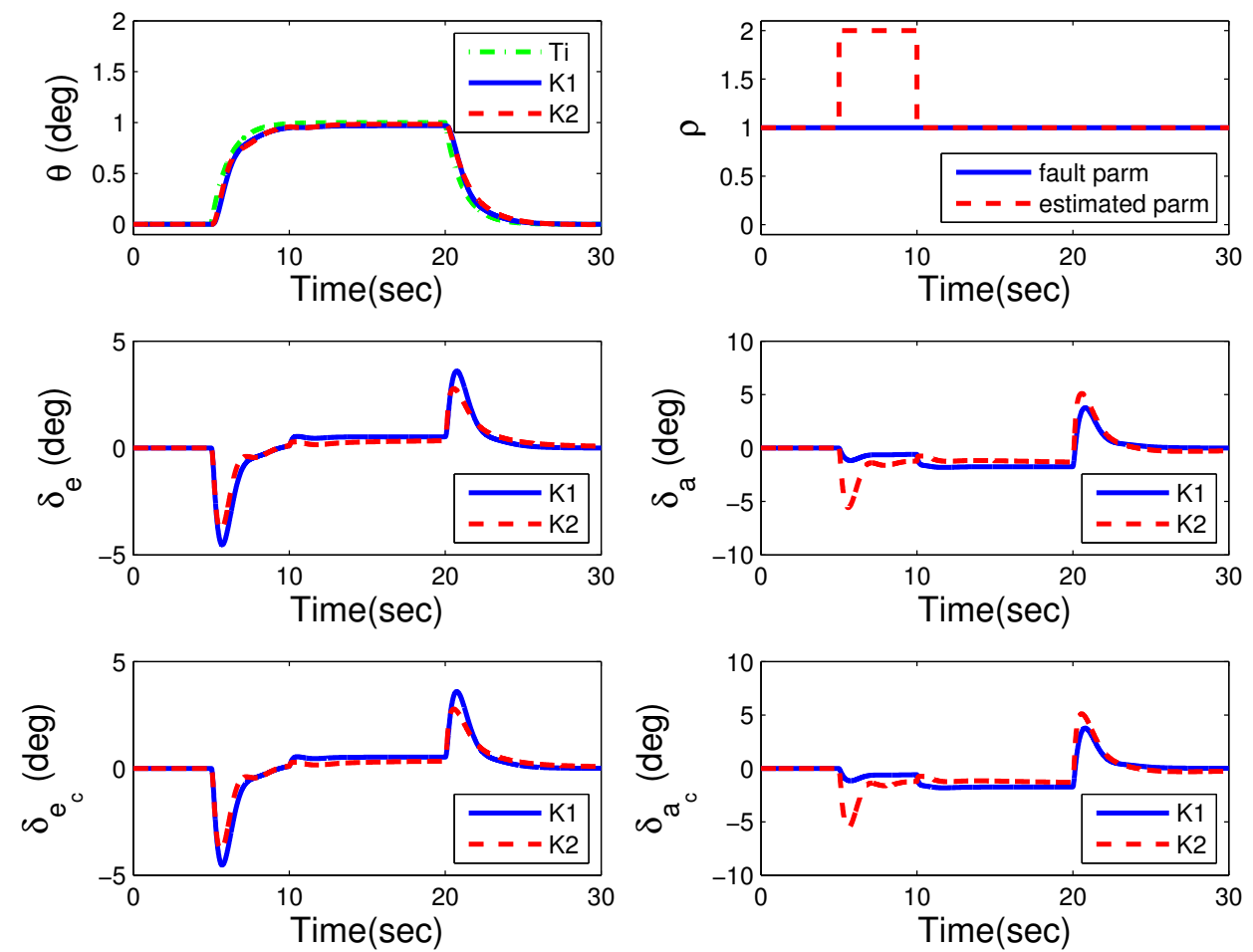

Figure 9: Time responses of the closed-loop system under the false identification case 3. 


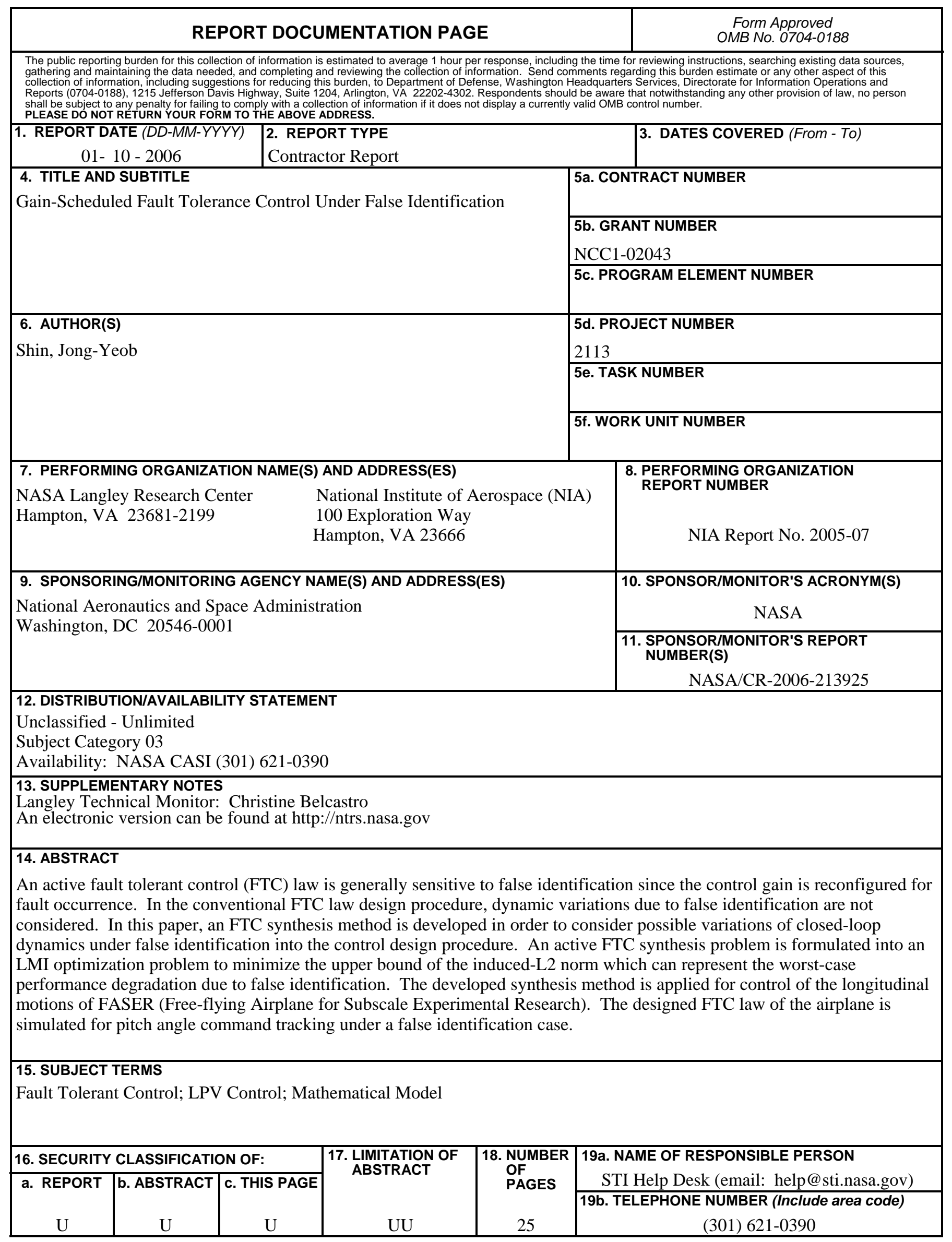

